\title{
The protective effect of curcumin against lithium-induced nephrotoxicity in rats
}

\author{
Mohammad Shaterpour ${ }^{1}$, Fatemeh Shaki ${ }^{1}$, Maryam Ghasemi $^{2}$, Majid Jafari-Sabet ${ }^{3}$, Ali Ziar ${ }^{1}$, Ramin Ataee ${ }^{1,4^{*}}$ \\ ${ }^{1}$ Pharmaceutical Sciences Research Center, Hemoglobinopathy Institute, Mazandaran University of Medical Sciences, Sari, Iran \\ ${ }^{2}$ Maryam Ghasemi, Immunogenetics Research Center, Mazandarn University of Medical Sciences, Sari, Iran \\ ${ }^{3}$ Department of Pharmacology, Iran University of Medical Sciences, Tehran, Iran \\ ${ }^{4}$ Thalassemia Research Center, Hemoglobinopathy Institute, Mazandaran University of Medical Sciences, Sari, Iran
}

Received: Feb 17, 2017, Revised: Jul 7, 2017, Accepted: Oct 21, 2017

\begin{abstract}
Lithium is an element which has been used as salts of chloride or carbonate for many years in the treatment of some psychological disorders such as mania, bipolar or schizophrenic diseases. Chronic application of lithium may induce some serious nephropathies such as natriuresis, renal tubular acidosis, tubulointerstitial nephritis progression to progressive chronic kidney disease and hypercalcemia and, most commonly, nephrogenic diabetes insipidus. Curcumin is an antioxidant derived from Curcuma longa (turmeric or curcuma) which has the ability to react directly with reactive species and up-regulation of many cytoprotective and antioxidant proteins. The preventive roles of curcumin in nephropathies were reported, but there was little information on the protective effect of curcumin against lithium-induced nephrotoxicity. In this study, male Wistar rats divided into five groups of six each and were treated as follows: group1; animals were received lithium chloride as $2 \mathrm{mmol} / \mathrm{kg}$, group 2; animals were received normal saline $(0,5 \%)$, group 3 ; animals were received curcumin $(200 \mathrm{mg} / \mathrm{kg}$ ), group 4 animals were received curcumin plus lithium and group 5; animals were received solvent intraperitoneally for three weeks. Then the animals were killed and biochemical parameters of blood were assayed and histopathological assessment was performed. The results have shown that curcumin significantly improved the biochemicals (BUN, creatinine, malondialdehyde). Curcumin prevented significantly the histological parameters that were changed by lithium administration in rats. Our results provide new insights into beneficial usages of curcumin in chronic nephrotoxicity induced by lithium salts.
\end{abstract}

Keywords: Lithium toxicity, curcumin, nephrotoxicity, lipid peroxidation, histopathology

Pharm Biomed Res 2017;3(2):33-38

\section{Introduction}

Lithium has been used clinically for almost 150 years. In the nineteenth century, Garrod and Hammond, suggested lithium salts for curing of gout and uric acid nephrolithiasis. In 1949, Cade reported highly successful results in 10 manic patients who received lithium salts (1). However, in the same year, Food and Drug Administration (FDA) withdrew the drug from the market because of the death of several patients due to lithium intoxication as heart failure or hypertension. Therefore, the use of lithium as a mood stabilizer progressed slowly and only in 1970, its use in the treatment of mania was approved by FDA (2). Since then litium has been widely used in the treatment of bipolar disorder. However it may induce renal toxicity which includes impaired urinary concentrating ability and natriuresis, renal tubular acidosis, tubulointerstitial nephritis progressing to chronic kidney disease and hypercalcemia. The most common adverse effect is nephrogenic diabetes insipidus which affects $20-40 \%$ of patients within weeks of lithium initiation. Chronic nephropathy may also be correlated with duration of lithium therapy (1).

Curcuma longa (turmeric or curcuma) is a plant member of the ginger family (Zingiberaceae), endemic in tropical and subtropical fields of India, China and South East Asia (2). Also it has been known that curcumin is a bifunctional antioxidant (3), because of its ability to react directly with reactive species and up-regulation of many cytoprotective and antioxidant proteins. Also it can scavenge superoxide anion $\left(\mathrm{O}_{2}^{-}\right)(4-5)$, hydroxyl radicals $(\mathrm{OH})(6), \mathrm{H}_{2} \mathrm{O}_{2}(4,6)$, singlet oxygen (7), nitric oxide $(8,9)$, peroxynitrite $(10)$ and peroxyl radicals (ROO2) (6). So these mechanisms can explain some of the cytoprotective effects of this material. The presence of phenolic groups in the structure of curcumin can explain its properties to react with reactive oxygen species (ROS) and reactive nitrogen species (RNS) and probably, one of the mechanisms through which it protects the epithelial cells of renal tubules from oxidative damage induced by $\mathrm{H}_{2} \mathrm{O}_{2}$ (11). The indirect antioxidant 
activity of curcumin is explained by its ability to induce the expression of cytoprotective proteins such as superoxide dismutase (SOD), catalase (CAT), glutathione reductase (GR), glutathione peroxidase (GPx), heme oxygenase 1 (HO-1) ,glutathione-S-transferase (GST), NAD(P)H; quinone oxidoreductase 1 (NQO1) and $\gamma$-glutamylcysteine ligase $(\gamma \mathrm{GCL})(2)$.

According to this background about antioxidant properties of curcumin and some reports which has described its role in nephrotoxicity by various chemicals, this research aimed to assay the effect of curcumin on lithium-induced chronic in rats by determining some indexes as lipid per oxidation of nephron tissues, pathological feauturs and BUN, cerateanine levels of animal serum.

\section{Materials and Methods}

Male Wistar rats $(\mathrm{n}=30,8$ weak-old), weighing 150-200 $\mathrm{g}$, were obtained from laboratory animal center of Mazandaran University of Medical Sciences. The rats were kept in an environmentally controlled room at constant temperature $\left(21 \pm 1{ }^{\circ} \mathrm{C}\right)$ and humidity $(75 \pm 5 \%)$ under a 12 $\mathrm{h}$ light/dark cycle. The animals were acclimatized for 1 week before the study and had free access to standard laboratory food and water ad labitum. The study has the permission of Ethics Review Committee for Ethics in Animal Experiments of the Mazandaran University of Medical Sciences and the guidelines for the Care and Use of Laboratory animals were strictly followed (Ethical Code:621).

Curcumin powder, haematoxylin and eosin and Malonyl dialdehyde Kit were all purchased from Sigma Co (Germany), lithium chloride and DMSO (Dimethyl sulphoxide) were provided from Scharlo (Spain) and BUN/creatinine Kit from Pars Azmoon (Iran).

\section{Experimental protocol}

According to similar studies on chronic nephrotoxicity in animals $(12,13)$, animals were divided into five groups of six:

Group 1: Six animals as negative control were received normal saline $0.9 \%$ intrapritoeanally for three weeks.

Group 2: Six animals as positive control were received lithium chloride $2 \mathrm{mmol} / \mathrm{kg}$ once a day intrapritoeanally for three weeks.

Group 3: Six animals treated were received curcumin dissolved in DMSO (200) $\mathrm{mg} / \mathrm{kg}$ intrapritoeanally 30 minutes after lithium chloride.

Group 4: Six animals were treated as group 3 but without lithium treatment.
Group 5: Six animals were received DMSO $0.5 \mathrm{ml} /$ day intrapritoeanally as solvent of curcumin.

After all treatments in day 22, the rats were sacrificed and blood samples were taken for serum analyses and the kidneys were removed for histological studies and lipid peroxidation test.

\section{BUN and creatinine analysis}

For determining BUN and creatinine, after killing of animal, blood was taken by syringe from their hearts and the plasma was collected by centrifuge 10000 RPM. BUN and creatinine were determined by Konelab 60i from Thermo Clinical Labsystems (ESP00, Finland) with kits that were supplied by Thermo Clinical Labsystems. The analyses and methodologies followed the instructions of the test kit manufacturer.

\section{Lipid peroxidation test}

Lipid peroxidation was estimated by thiobarbituric acid (TBA) reaction with malondialdehyde (MDA), which the latter is the product formed from membrane lipid peroxidation (15). In brief, after homogenization of animals' kidneys with homogenizer in Tris buffer (15), 2.5 $\mathrm{mL}$ homogenate, $0.5 \mathrm{~mL}$ of $0.9 \% \mathrm{NaCl}$, and $1.0 \mathrm{~mL}$ Trichloroacetic acid (TCA $20 \% \mathrm{w} / \mathrm{v}$ ) were added into the mixture. The mixture was then centrifuged for 20 minutes at $4000 \mathrm{~g}$ at $4{ }^{\circ} \mathrm{C}$. $0.25 \mathrm{~mL}$ TBA reagent was added to 1.0 $\mathrm{mL}$ supernatant and the mixture was incubated at $95{ }^{\circ} \mathrm{C}$ for $1 \mathrm{~h}$ and cooled under running tap water prior to addition of $1 \mathrm{~mL} n$-butanol. After thorough mixing, the mixture was centrifuged for 15 minutes at $4000 \times \mathrm{g}$ at $4{ }^{\circ} \mathrm{C}$. The organic layer was transferred into a clear tube and the absorbance was measured at $532 \mathrm{~nm}$ with a spectrophotometer (UV1700). The rate of lipid peroxidation was expressed as $\mu$ moles of MDA formed/gram wet weight of the tissue.

\section{Histopathological analysis}

For histopathological analysis, after preparing the sections from kidney tissues of rats and embedding in paraffin block, preparation of section with microtome, and staining with haematoxylin and eosin protocol, the slides were observed by light microscope with magnificence of 10 and their pathological changes were assessed.

\section{Statistical analysis}

The comparisons between groups for biochemical and lipid peroxidation markers, were analyzed by one-way ANOVA and t-test by (SPSS software, Chicago, USA) and histopathological analysis were done qualifiactionally. 


\section{Results}

\section{BUN and creatinine test}

According to biochemical analysis, BUN and creatinine in lithium groups have been increased, (mean of BUN: 55.4 $\mathrm{mg} / \mathrm{dl}$ and creatinine: $0.58 \mu \mathrm{g} / \mathrm{dl})$. BUN and creatinine levels were diminished in curcumin + lithium group (mean of BUN: $37 \mathrm{mg} / \mathrm{dl}$ and creatinine: 0.39$)$, significantly $(P<$ 0.01) (Fig1 and 2).

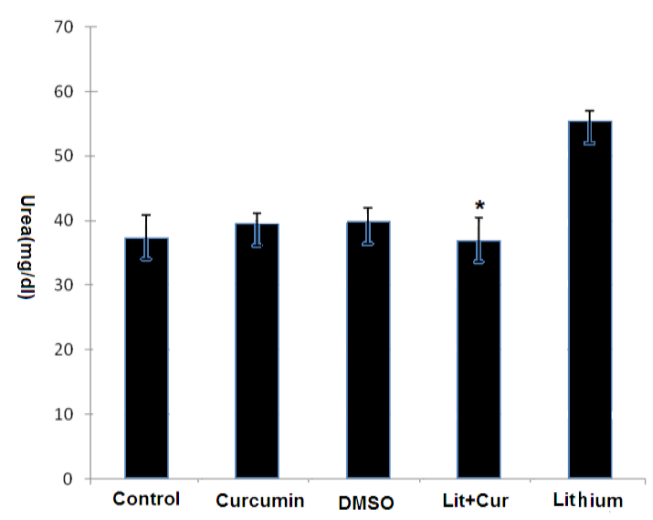

Figure 1 Comparison between serum concentration of urea (BUN), between groups after various types of drug treatments * : significant, compare with lithium group $P<0.01$.

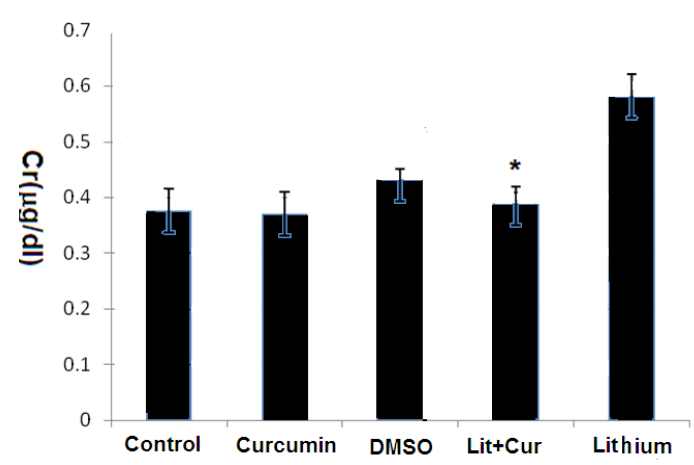

Figure 2 Comparison between serum concentration of creatinine $(\mathrm{Cr})$, after various types of drug treatments *: significant, compare with lithium group $\mathrm{P}<0.01$, Cur (curcumin).

\section{Lipid peroxidation results}

Malondialdehyde as an index of lipid peroxidation has been increased in lithium group $(1.07 \mu \mathrm{M} / \mathrm{gr})$ and diminished in curcumin + lithium group $(0.72 \mu \mathrm{M} / \mathrm{gr})$ significantly $(P$ $<0.01$ ) (Fig. 3, Fig. 4).

\section{Pathological results}

In lithium group, dilation, oedema and inflammations of microtubules of nephrons and focal and moderate lymphocytic infiltration in interstitial tissues showed (were indicative of) inflammatory reactions (Fig 5(A, B)); but

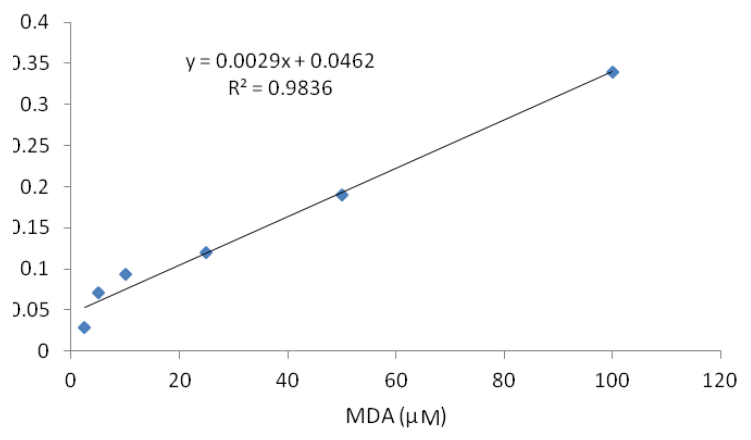

Figure 3 Lipid peroxidation curve after various chemical treatments

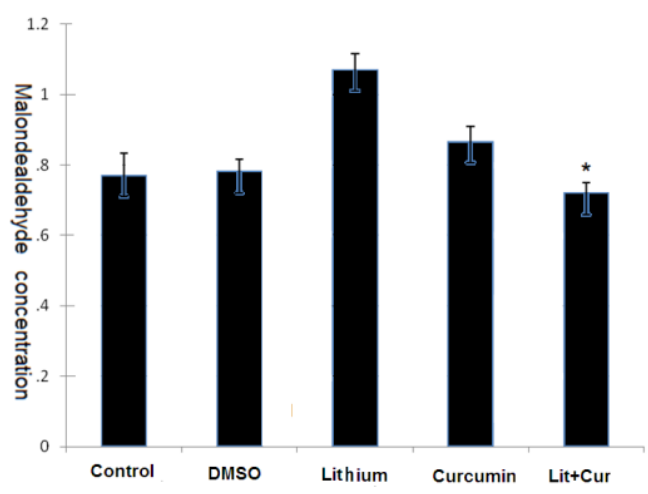

Figure 4 Lipid peroxidaton analysis of rats' kidney tissues after treatments with different types of chemical treatments, MDA as lipid peroxidation indexed, detemined as $\mu \mathrm{M} /$ gram weight of the tissue. *: significant, compare with lithium group $\mathrm{P}<0.01$, Cur (curcumin).

with specific staining (periodic acid shift and three chrome), the gloumerols were unchanged (Fig 5C) which indicates that the filtration ability of nephrons was not disturbed seriously. In lithium + curcumin group, dilation and inflammations of tubules were improved clearly (Fig 5D) but yet, mild lymphocytic infiltration in interstitial were observed (Fig 5E) and as lithium group the gloumerols were unchanged.

\section{Discussion}

The results of recent studies showed, chronic treatment with lithium chloride at dose of $2 \mathrm{mmol} / \mathrm{kg}$ has induced oxidative stress, increased BUN and creatinine of blood and inflamed the microtubules. Also curcumin could have ameliorated BUN and creatinine levels and improved histopathological defects following lithium chronic nephrotoxicity. It significantly prevented the lipid peroxidation (as an index of oxidative stress) in nephron tissues. As we know BUN and creatinine can be an important indicator of the kidney function (14). Mechanism of lithium toxicity may be due to increase in expression of cyclooxygenase-2 and in urinary prostaglandin E2 


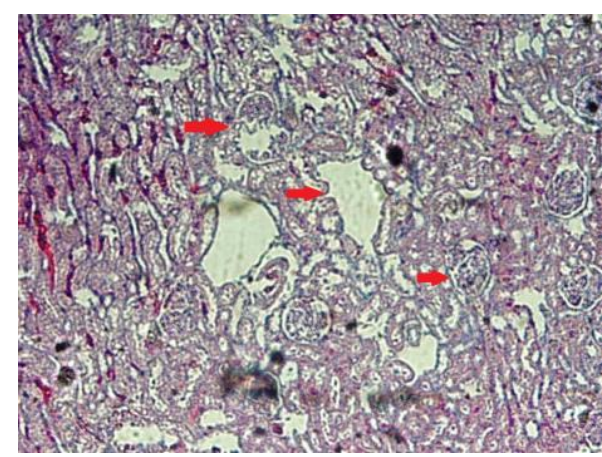

A)

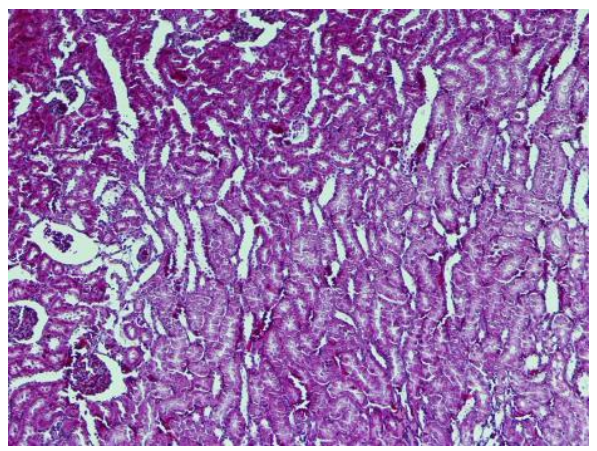

C)

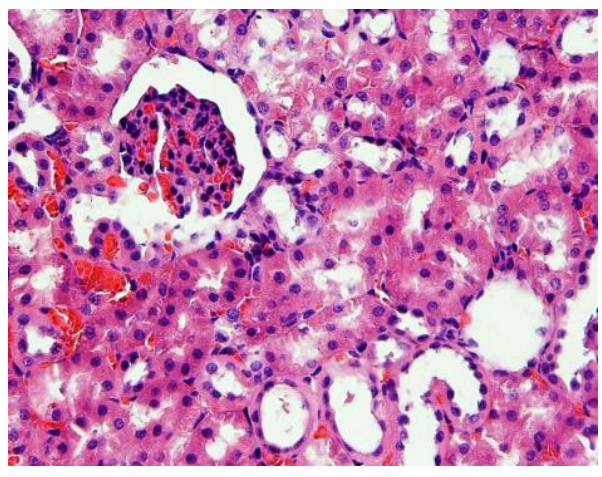

E)

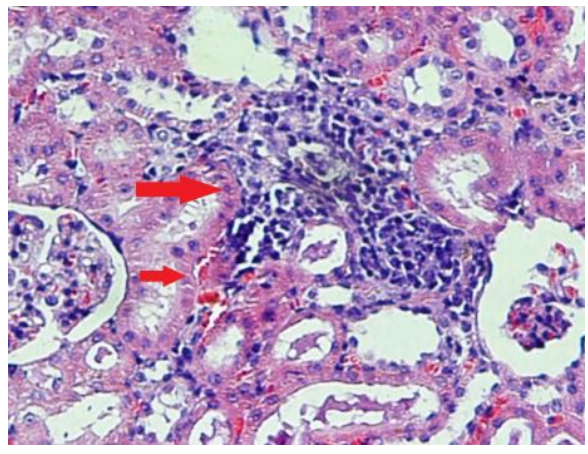

B)

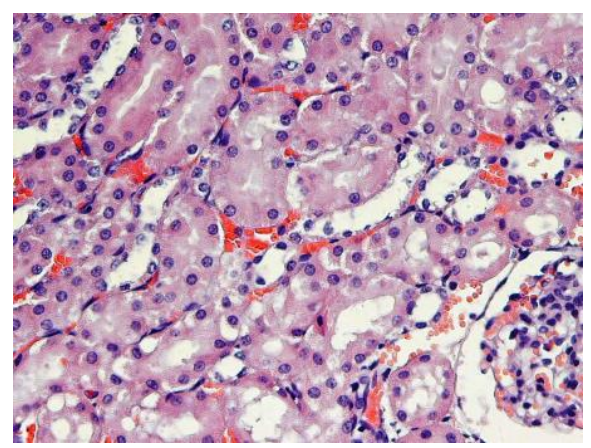

D)

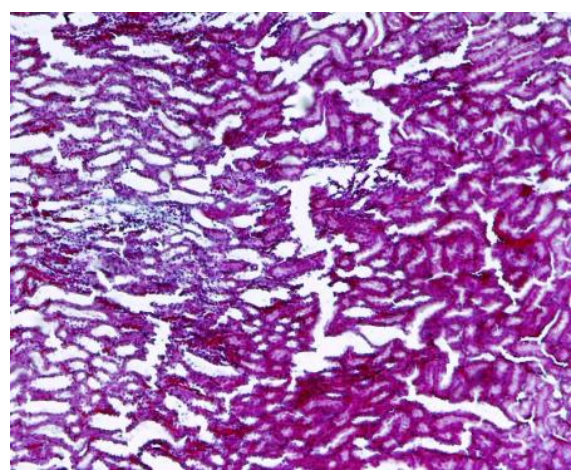

F)

Figure 5 Histopathological results after different chemical treatments A) Dilation, edema and inflammations of tubules in lithium group, B) Focal and moderate lymphocytic infiltration in interstitial tissues in lithium group; C) Unchanged gloumerols in lithium group, stained with PAS and Three Chrome ; D) Reduced in dilation and inflammations of tubules in Lithium+curcumin; E) Mild lymphocytic infiltration in interstitial tissues in lithium+curcumin group and F) Unchanged gloumerols in Lithium +curcumin group, stained with PAS and Three Chrome

excretion by modularly interstitial cells (15). These prostaglandins then act on principal cells to induce lysosomal degradation of AQP2 water channels and to decline urine concentrating ability. Lithium may reduce $A Q P 2$ gene transcription, an effect that is prostaglandin independent, leading to a further decrease in concentrating ability (15). Lithium induces remodeling of collecting duct which is characterized by a decreased population of principal cells relative to the number of intercalated cells, a phenomenon that was previously presumed to be due to apoptosis $(17,18)$. However, lithium may actually lead to proliferation of principal cells, which then will undergo cell cycle arrest (18). This happening may also be responsible for the development of interstitial nephritis and renal fibrosis. Aquaporin-2 (AQP2) is the vasopressin-regulated water-channel protein expressed at the connecting tubule and collecting duct and plays an important role in urine concentration and body-water homeostasis through 
short-term and long-term regulation of collecting duct water permeability. The signaling transduction pathways result in the AQP2 trafficking to the apical plasma membrane of the collecting duct principal cells, including AQP2 phosphorylation, RhoA phosphorylation, actin depolymerization, and calcium mobilization. Dysregulation of AQP2 has been shown to be importantly associated with a number of clinical conditions characterized by body-water balance disturbances, including hereditary nephrogenic diabetes insipidus (NDI) and lithium-induced NDI (18).

Previous studies have reported several preventive roles for curcumin in nephrotoxicity. Curcumin treatment in an animal model of diabetic nephropathy has attenuated proteinuria and improved creatinine clearance after three weeks (2). In addition, it decreased oxidative stress by reducing levels of subunits of nicotinamide adenine dinucleotide phosphate NADPH (2). It was supposed that the renoprotective effect of curcumin was related to the downregulation of the profibrotic cytokines as vascular endothelial growth factor (VEGF), TGF-B, CTGF and osteopontin as well as in extracellular matrix proteins, fibronectin and collagen IV(1). Cellular events such as inhibition of NF- $\mathrm{KB}$ (19-21) and decrease of macrophage's infiltration, histone acetyltransferase p300 protein and diminishing of oxidative stress are among the reported mechanisms (19-21) through which curcumin can prevent nephropathies.Waseem et al. has shown that curcumin has protective effect against acute nephrotoxicity model induced with cisplatin in rat and it could have reduced lipid peroxidtion and proteinyl carbonyl levels (22). Ukei et al. has shown that levels of inflammation factors as tumor necrosis factor (TNF)- $\alpha$ and monocyte chemoattractant protein (MCP)-decreased in mice which received curcumin, $10 \mathrm{mg} / \mathrm{kg}$ with cisplatin (23). Manikandan et al. reported that curcumin could reduced iNOS and NF-қB expression (19) in acute nephrotoxicity model of gentamyci and curcumin administration reduce BUN, creatinine and MDA in rats in model of acetaminophen induced nephrotoxicity (24). Our study is very similar to Cekmen exam with similar MDA and histopatholgical results and in parallel with some others studies as Ukei M et al who has shown suppressing effect of curcumin against inflammation factors as (TNF)- $\alpha$ with curcumin in nephrotoxicity.

\section{Conclusion}

This study was one of the first studies which showing the preventive role of curcumin against lithium chronic nephrotoxicity. As curcumin is a safe additive material with no drug interaction, it can be suggested for co-treatment with Lithium in various psychological disorders, However more complementary in vivo studies are needed to assay the effect of curcumin on more parameters in oxidative stress.

\section{Acknowledgments}

The authors would like to appreciate the staff of pathology department at Bou Ali hospital for preparation and staining of histological slides and also Dr. Jamshid Yazdani for his nice collaboration on statistical analysis.

\section{Conflict of interest}

There is not any conflict of interest between authors in all part of this study.

\section{References}

1. Oliveira JL, Silva Júnior GB, Abreu KL, Rocha Nde A, Franco LF, Araújo SM, Daher Ede F. Lithium nephrotoxicity. Rev Assoc Med Bras 2010;56:600-6.

2. Trujillo J, Chirino YI, Molina-Jijón E, Andérica-Romero AC, Tapia E, Pedraza-Chaverrí J. Renoprotective effect of the antioxidant curcumin: Recent findings. Redox Biol 2013;1:448-56.

3. Dinkova-Kostova AT, Talalay P. Direct and indirect antioxidant properties of inducers of cytoprotective proteins. Mol Nutr Food Res 2008;52:128-38.

4. Ak T, Gülçin I. Antioxidant and radical scavenging properties of curcumin. Chem Biol Interact 2008;174:27-37.

5. Sreejayan N, Rao MN. Free radical scavenging activity of curcuminoids. Arzneimittelforschung 1996;46:169-71.

6. Barzegar A , Moosavi-Movahedi Ali A .Intracellular ROS Protection Efficiency and Free Radical-Scavenging Activity of Curcumin. PLoS ONE 2011; 6: e26012:1-7

7. Das KC, Das CK. Curcumin (diferuloylmethane), a singlet oxygen ((1)O(2)) quencher. Biochem Biophys Res Commun 2002 ;295:62-6.

8. Sreejayan Rao MN. Nitric oxide scavenging by curcuminoids. J Pharm Pharmacol 1997;49:105-7.

9. Sumanont Y, Murakami Y, Tohda M,Vajragupta O, Matsumoto $\mathrm{K}$, Watanabe $\mathrm{H}$. Evaluation of the nitric oxide radical scavenging activity of manganese complexes of curcumin and its derivative. Biol Pharm Bull 2004;27:170-3.

10. Kim JE KA, Chung HY, Han SY, Kim Choi JS. In vitro peroxynitrite scavenging activity of diarylheptanoids from Curcuma longa. Phytother Res 2003;17:481-4.

11. Augustyniak A, Bartosz G, Cipak A, Duburs G, Horáková L, Luczaj W, et al. Natural and synthetic antioxidants: an updated overview. Free Radic Res 2010;44:1216-62.

12. Srimuangwong K, Tocharus C, Tocharus J, Suksamrarn A, Chintana PY. Effects of hexahydrocurcumin in combination with 5-fluorouracil on dimethylhydrazine-induced colon cancer in rats. World J Gastroent 2012;21:6951-9.

13. Tirkey N, Kaur G, Vij G, Chopra K. Curcumin, a diferuloylmethane, attenuates cyclosporine induced renal dysfunction and oxidative stress in rat kidneys. BMC Pharmacol 2005;5:1-10.

14. Bas M, Tugcu V, Kemahli E, Ozbek E, Uhri M, Altug T, Tasci AI. Curcumin prevents shock-wave lithotripsy-induced renal injury through inhibition of nuclear factor kappa-B and inducible nitric oxide synthase activity in rats. Urol Res 2009;37:159-64.

15. Kortenoeven ML, Schweer H, Cox R. Lithium reduces aquaporin-2 transcription independent of prostaglandins. Am J Physiol Cell Physiol 2012;302:131-40. 
16. Ecelbarger CA. Lithium treatment and remodelling of the collecting duct. Am J Physiol Renal Physiol 2006;291:37-8.

17. Christensen BM, Kim YH, Kwon TH, Nielsen S. Lithium treatment induces a marked proliferation of primarily principal cells in rat kidney inner medullary collecting duct. Am J Physiol Renal Physiol 2006;291: 39-48.

18. De Groot T, Alsady M, Jaklofsky M, Otte-Höller I, Baumgarten R, Giles RH, et al. Lithium causes $\mathrm{G} 2$ arrest of renal principal cells. J Am Soc Nephrol 2014; 25:501-10.

19. Kwon T, Frøkiær J, Nielsen S. Regulation of aquaporin-2 in the kidney: A molecular mechanism of body-water homeostasis. Kidney Res Clin Pract 2013; 32: 96-102.

20. Chiu J, Khan ZA, Farhangkhoee H, Chakrabarti S. Curcumin prevents diabetes-associated abnormalities in the kidneys by inhibiting p300 and nuclear factor-kappaB. Nutrition 2009;25:964-72.
21. Soetikno V, Sari FR, Veeraveedu PT, Thandavarayan RA, Harima M, Sukumaran V, et al. Curcumin ameliorates macrophage infiltration by inhibiting NF-kB activation and proinflammatory cytokines in streptozotocin induced-diabetic nephropathy. Nutr Metab 2011;8:35.

22. Waseem M, Kaushik P, Parvez S. Mitochondria-mediated mitigatory role of curcumin in cisplatin-induced nephrotoxicity. Cell Biochem Funct 2013;31:678-84.

23. Ueki M, Ueno M, Morishita J, Maekawa N. Curcumin ameliorates cisplatin-induced nephrotoxicity by inhibiting renal inflammation in mice. J Biosci Bioeng 2013;115:547-51.

24. Manikandan R, Beulaja M, Thiagarajan R, Priyadarsini A, Saravanan $\mathrm{R}$, Arumugam M. Ameliorative effects of curcumin against renal injuries mediated by inducible nitric oxide synthase and nuclear factor kappa B during gentamicin-induced toxicity in Wistar rats. Eur $\mathbf{J}$ Pharmacol 2011;670:578-85. 